\title{
Performance Analysis of Adaptive Location Update Schemes for Continuous Cell Zooming Algorithm in Wireless Networks
}

\author{
Khin Cho Tun ${ }^{1, a}$ and Kunagorn Kunavut ${ }^{2, b, *}$ \\ 1 Department of Electronic Engineering, Mandalay Technological University, Mandalay, Myanmar \\ 2 Department of Communication and Computer Network Technology, Vincent Mary School of Science \\ and Technology Assumption University, Thailand \\ E-mail: akhinchotun@gmail.com, bkkunagorn@gmail.com (Corresponding author)
}

\begin{abstract}
To reduce the transmitted power of base stations in mobile wireless networks, continuous cell zooming algorithm is a feasible dynamic cell zooming algorithm. In this algorithm, location management is required in order to know the locations of users. Movement-based Update is not compatible and the application of Convention Periodic Update (CPU) scheme in continuous cell zooming algorithm can lead to a high signaling cost. Thus, aiming to highlight the effectiveness of newly proposed location update schemes, Time-Adaptive Periodic Update (TAPU) and Location-Adaptive Periodic Update (LAPU), a simulation-based performance analysis is conducted. Applying in continuous cell zooming algorithm, the performances of TAPU and LAPU are compared to that of Convention Periodic Update (CPU) scheme in terms of transmitted power ratio, outage ratio and the number of update messages. The performances of TAPU and LAPU are analyzed in a network with different number of users and in a network with different average moving speeds of users. The results show that compared to CPU, both TAPU and LAPU have no significant effect on power saving capability of continuous cell zooming algorithm in every scenario. Meanwhile, LAPU and TAPU give a significant reduction of update messages in every scenario. In terms of QoS effect, LAPU gives approximately the same outage ratio as CPU and a higher outage ratio occurs in TAPU.
\end{abstract}

Keywords: Location update, cell zooming, power saving, wireless networks.

ENGINEERING JOURNAL Volume 20 Issue 1

Received 17 March 2015

Accepted 7 July 2015

Published 29 January 2016

Online at http://www.engj.org/

DOI:10.4186/ej.2016.20.1.137 


\section{Introduction and Motivation}

Today, the improvement of energy efficiency of wireless communication networks has become a critical topic of discussion to cope with the escalating costs of energy in our society [1]. Most of researchers try to improve performance in wireless networks $[2,3,4]$. For the goal of improving energy efficiency of the wireless communication networks, many different approaches (see in [1]) have been proposed to manage the base stations, which are the most energy-greedy components in a particular wireless network. Here, "cell zooming" has been considered as one of the potential approaches for energy-efficient operation of the base stations. In a few words, cell zooming is a technique to adjust the transmitted power of base stations according to the traffic condition in each cell and it can give advantages of power-saving and/or traffic load-balancing in the network [5]. On the other hand, the QoS (Quality of Service) can be degraded due to cell zooming. Since then, many researchers proposed a number of feasible cell zooming algorithms that satisfied best power-saving and the constrained QoS [6]. A common notice from some previous works was [7-12] that it is necessarily important to know the traffic spatial distribution in the network in order to perform more energy-efficient cell zooming while maintaining the compromised QoS. At the same time, the challenge given was how to cope with the requirement of massive information exchange in search of traffic spatial distribution.

Recently, the authors of [12] developed and evaluated state-of-the-art dynamic cell zooming algorithms called discrete zooming, fuzzy zooming and continuous zooming. In these cell zooming algorithms, location-management strategies are required in order to know the traffic spatial distribution which is essential information to perform efficient cell zooming. Then, the continuous cell zooming algorithm showed a better performance in terms of power saving capability compared to fuzzy and discrete cell zooming algorithms (see in $[12,13])$. According to the concept of continuous cell zooming algorithm, periodic location update scheme is the most feasible location management strategy to know the user location for cell zooming operation [13]. However, unfavorable outcome from using periodic update in continuous cell zooming algorithm is that user could experience outage (the effect on the QoS) due to its mobility during cell zooming period. On one hand, the outage can be reduced by using a shorter update interval. However, on the other hand, it leads to a high signaling cost because all users need to update very frequently. Thus, how to minimize the requirement of massive information exchange between BSs and users has become the major issue to increase the potentiality and efficiency of continuous cell zooming algorithm.

After surveying cost-effective location-management strategies proposed in the literature [14-26], we gained the following facts. First, most newly proposed location-management strategies were based on conventional movement-based methods. Second, number of boundary crossing or distance of movement was applied as an alarm for mobile terminal to update. Third, daily itinerary (profile of movement) of each user is importantly required to develop a more efficient location-management strategy. Fourth, time-based location update methods were given less attention. Scarcely, the authors of [27] proposed a time-based update method in which the location update performed by the user was based on user activity and on the load factor, computed by the network. Here, each cell in the network computes its own load factor, independent of other cells, and announces to the users as a broadcast message, through the downlink control channel. According to the load factor of BS and its activity, each user decide whether it should update or not at each interval. Then, Samir et al. [28] proposed co-operative location update algorithm in which a representative user collects update messages of neighboring users and transmits all information to the base station along with its own location information at every update interval. However, these timebased methods are not compatible with continuous cell zooming algorithm. Thus, a compatible and effective time-based location update method is currently in demand to integrate as a mechanism in continuous cell zooming algorithm.

For this reason, we have proposed two new location update schemes, namely; Time-Adaptive Periodic Update (TAPU) and Location-Adaptive Periodic Update in our recent work [29].The proposed location update methods were used in continuous cell zooming algorithm. The simulated results showed that compared to Conventional Periodic Update (CPU) scheme, the TAPU and LAPU schemes could reduce about half of total number of update messages in cell zooming while they were keeping the same power capability of continuous cell zooming algorithm. These newly proposed update schemes techniques are effective and very challenging, and then they are required to be analyzed in different scenarios. However, the scope of performance analysis on proposed update schemes is still limited and the information provided in our previous attempt [26] is still inadequate. In this regard, the objective of this paper is to 
address the performances of TAPU and LAPU in a larger scope with the variation of influential parameters such as number of users in the network and average moving speed of the users, aiming at providing more detailed information and further understanding.

The rest of the paper is organized as follows. The continuous cell zooming algorithm is briefly explained in next section. The proposed location update schemes, TAPU and LAPU, are described in section 3. Next, simulation methodology is explained in section 4 . Then, results and discussion are given in section 5 which is followed by conclusion.

\section{Continuous Cell Zooming Algorithm}

Although, the concept of continuous cell zooming algorithms can be found in previous works [5, 6, 29], it is worth to recall here for sequential understanding. Figure 1 illustrates the concept of continuous cell zooming algorithm. In this algorithm, the location of the farthest user in each cell is detected for each cell zooming period by means of location management. Then, the transmitted power is adjusted to the distance that accommodates the farthest user in the cell rather than transmitting to the original cell edge regardless of user location. The required power to transmit signal to the farthest users is adjusted as follows:

$$
\frac{P_{T x z}}{P_{T x i}}=\left(\frac{R_{z}}{R_{i}}\right)^{n}
$$

In Eq. (1), $P_{T x x}$ is power required to transmit the signal to zoomed cell edge, $P_{T x i}$ is power required to transmit the signal to original cell edge, $R_{z}$ denotes the radius of zoomed cell edge, $R_{i}$ represents the radius of original cell edge and $n$ is the path loss exponent. Then, this cell zooming algorithm has no switch-off scheme of BSs to avoid the effect of delay in on/off switching and only transmitted power of BSs is adjusted individually according to traffic condition in each cell.

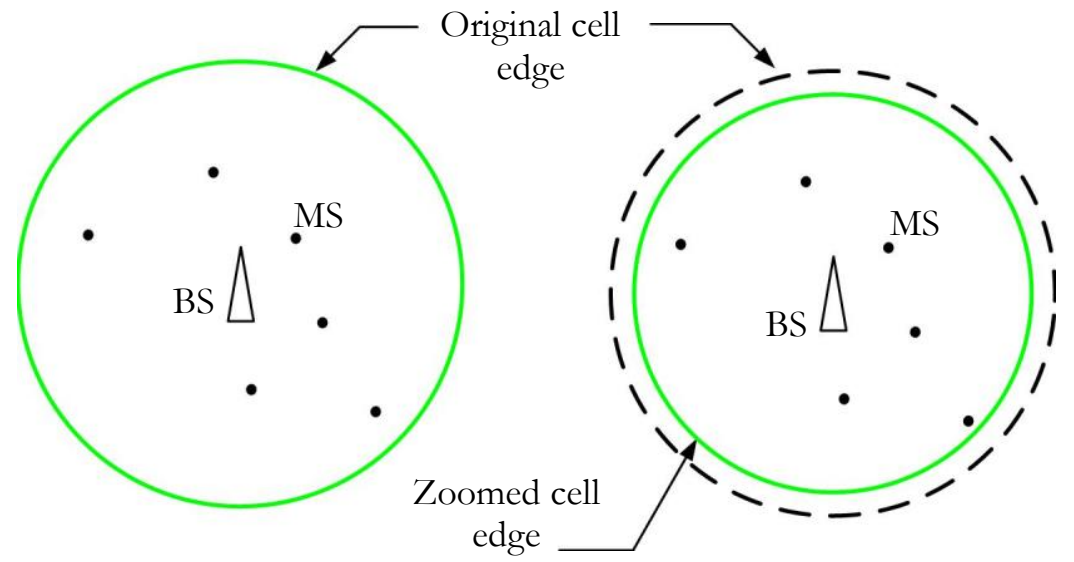

Fig. 1. Demonstration for continuous cell zooming.

\section{Proposed Location Update Schemes}

As stated in continuous cell zooming algorithm, the locations of users in each cell must be known to perform cell zooming. To know the locations of the users, location update process is necessarily important. Since the existing location update schemes are less compatible and effective, the following feasible location update schemes were proposed in our previous work [29].

\section{1. $\quad 3.1$ Time-Adaptive Periodic Update (TAPU) Scheme}

In time-adaptive periodic update (TAPU) scheme, the periodic update interval is varied according to general user mobility level that changes with local time. For example, the number of moving users at night hours is generally far smaller than that in day time. In day time, the numbers of moving users in officestarting hours (morning hours), lunch time (mid-day hours) and office-ending hours (evening hours) are 
larger than that in office-working hours. Therefore, a longer update interval can be set for hours during which user mobility level is low and a shorter update interval can be set for hours of higher mobility level. As a sample case in this work, three different user mobility levels (such as Level-1 < Level-2< Level-3) are assumed for seven different local time intervals. It is shown in Fig. 2(a). It should be noted that the pattern of user mobility level can be different from network to network and from office-day to holiday.

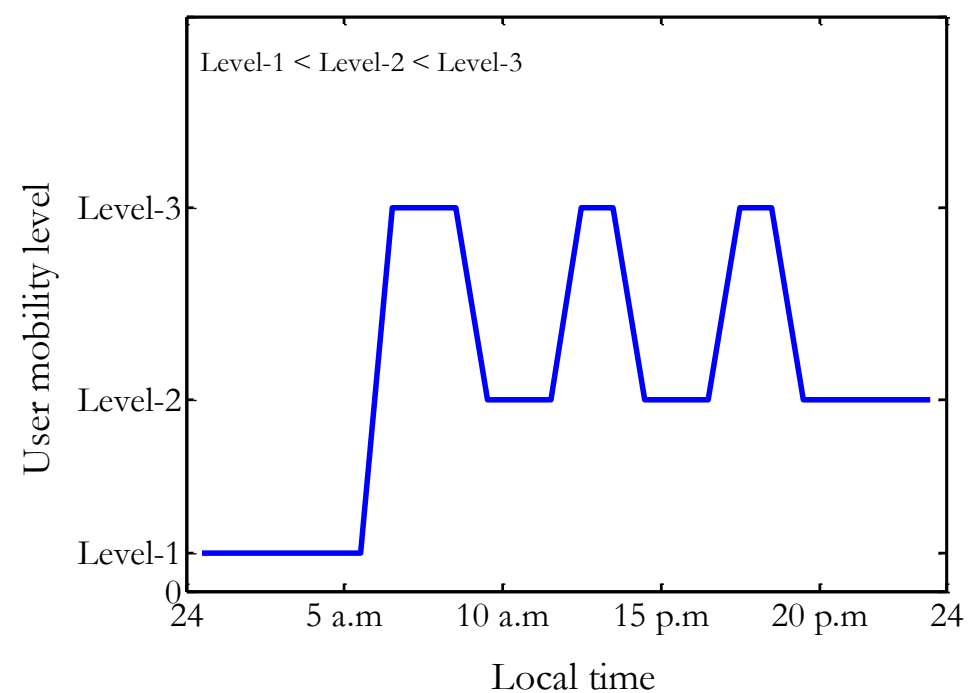

(a)

\section{For BS (network)}

- Select $\Delta t_{u p 1}, \Delta t_{u p 2}, \Delta t_{u p 3}$ such as $\Delta t_{u p 1}>\Delta t_{u p 2}>\Delta t_{u p 3}$

- Inspect local time to estimate corresponding user mobility level

- Set appropriate update interval for that time

Set $\Delta t_{u p 1}$ for mobility Level-1

Set $\Delta t_{u p 2}$ for mobility Level-2

Set $\Delta t_{u p 3}$ for mobility Level-3

- Broadcast to all users to do update with specified update interval

- Reset zoomed cell edge $\left(R_{*}\right)$ to original cell edge $\left(R_{i}\right)$ for next update process

- Receive $d_{M S}$ from $\forall$ MSs in update process

- Find $\max \left[d_{M S}\right]$

- Perform cell zooming by setting $R_{z}=\max \left[d_{M S}\right]$ for specific update interval

\section{For MS}

- Listen to network to obtain specified update interval

- Measure $d_{M S}$ by means of pilot signal strength at every period

- Update location when update time arrives

(b)

Fig. 2. Time-adaptive periodic update (TAPU): (a) a sample user mobility level assumption in a day; (b) pseudo code of TAPU.

The TAPU scheme is shown in Fig. 2(b). For local time intervals that have three different user mobility levels, three different update intervals will be used. These update intervals must be defined prior to the cell zooming operation and they are adjustable to suite with the network environment (such as user mobility level). Then, an appropriate update interval is statically selected for a local time period (for a mobility level). However, in the future work, it may be integrated with the intelligent system to learn/predict user mobility in order to dynamically define these time interval. Here, $\Delta t_{u p 1}$ is the longest update interval, $\Delta t_{u p 2}$ is medium 
update interval and $\Delta t_{u p}$ is the shortest update interval. Then, the network will broadcast to all users to perform update by using the selected update interval. Then, it will start update process by resetting the current zoomed cell edge $\left(R_{\ni}\right)$ to original cell edge $(R \imath)$ for a moment for update process to cover the outage MSs in previous period (if any). Therefore, all users will update and the network will receive update messages that contains location information of each MS. Then, the network will find out the farthest user in the cell by analyzing all messages. Then, it will perform cell zooming by setting the new farthest user location as new zoomed cell edge for that period. In this procedure, the network will perform receiving update messages and cell zooming alternatively.

The work of MS is to often listen to the network to know the update interval. Then, it has to measure its distance $\left(d_{M S}\right)$ from BS at each update interval according to pilot signal strength. When update time arrives, each MS has to report its location $\left(d_{M S}\right)$ to the BS so that network can analyze the location of the farthest user in each cell. Here, it should be noted that the values of update intervals used in TAPU are configurable as the network administrator desires according to the time-dependent mobility levels in the corresponding network.

\subsection{Location-Adaptive Periodic Update (LAPU) Scheme}

The second scheme is location-adaptive periodic update (LAPU) scheme in which the users perform location update according to their locations. For this scheme, each cell is logically divided into two regions. The outer region is defined as 'update region or update area' (see in Fig. 3(a)). At every update period, all users in update region are supposed to perform update and the other users outside update region are supposed to skip updating their locations. The concept of LAPU can be explained by using Fig. 3(a). In Situation I, only users A and B will update in coming period since they are in update region. But the user C will not update. In Situation II, there is no user in update region and the network will receive no update message. In such a situation, the network will inform all the users to update their locations. Then, the cell will be shrunk to the new farthest user in coming period.

Then, as shown in Fig. 3(a), the update area is bounded by the original cell edge $\left(R_{i}\right)$ and the inner radius $\left(R_{b}\right)$. In this work, the update area is dynamically changed by changing the inner radius $\left(R_{b}\right)$ with respect to the zoomed cell edge. The inner radius $\left(R_{b}\right)$ for next period is shorter than zoomed cell radius $(R z)$ and it is configurable. It is effective to avoid Situation II explained above as much as possible. The information of zoomed cell edge $\left(R_{\vartheta}\right)$ is broadcasted to all users in every cell zooming period.

The pseudo code of LAPU scheme is shown in Fig. 3(b). In LAPU scheme, the task of the network is to set a periodic update interval $\left(\Delta t_{u p}\right)$ for all users uniformly. Then, it has to regularly receive update messages from users in specified update region. If there is no update message, the network realizes that there is no MS in update region and all MSs are inside non-update region. For that situation, the BS has to inform all users in its cell to perform update to know the farthest user location for that period even though they are not in update region. After receiving the update messages, the network will analyze them to find out the location of the farthest user. Then, it will perform cell zooming by setting the new farthest user location as new zoomed cell edge for that period. Also, the network has to broadcast the information of current zoomed cell edge to all users to evaluate the update region in next period. In this procedure, the network will perform receiving update messages and cell zooming alternatively.

The task of MS is to listen to the network at every period to know the pilot signal strength threshold $\left(\lambda_{b}\right)$ that that is correlated to the inner radius $\left(R_{b}\right)$ of update region. It has to constantly keep the pilot signal strength threshold $\left(\lambda_{i}\right)$ that represents $\left(R_{i}\right)$. Then, it has to measure the distance $\left(d_{M S}\right)$ from BS according to pilot signal strength at every period. Moreover, it has to inspect its location whether it is between $R_{b}$ and $R_{i}$. If it is inside update region, it has to perform update. Otherwise it can skip update.

Here, one thing to be discussed is how a user senses the update region. In practice, the common pilot channel $(\mathrm{CPICH})$ signal is used by mobile terminals to sense the cell boundary so that it can do cell reselection and handover [30-32]. This capability of user premise can be exploited to sense the update region in LAPU scheme by using two pilot signal thresholds $\left(\lambda_{b}\right.$ and $\left.\lambda_{i}\right)$ that are correspondent to the inner radius of update region $\left(R_{b}\right)$ and original cell edge $\left(R_{i}\right)$. Also, there are a number of potential userpositioning techniques although they are still under research [33]. It should be noted that the location reported by mobile terminal is only the distance from BS but not global coordinates. Thus, it will not break the privacy of the user. Although it is arguable that there can be accuracy problem in user-positioning 
technique by means of pilot signal strength, it is beyond the scope of this work since this work is aimed to highlight the potentiality of adaptive periodic update schemes.

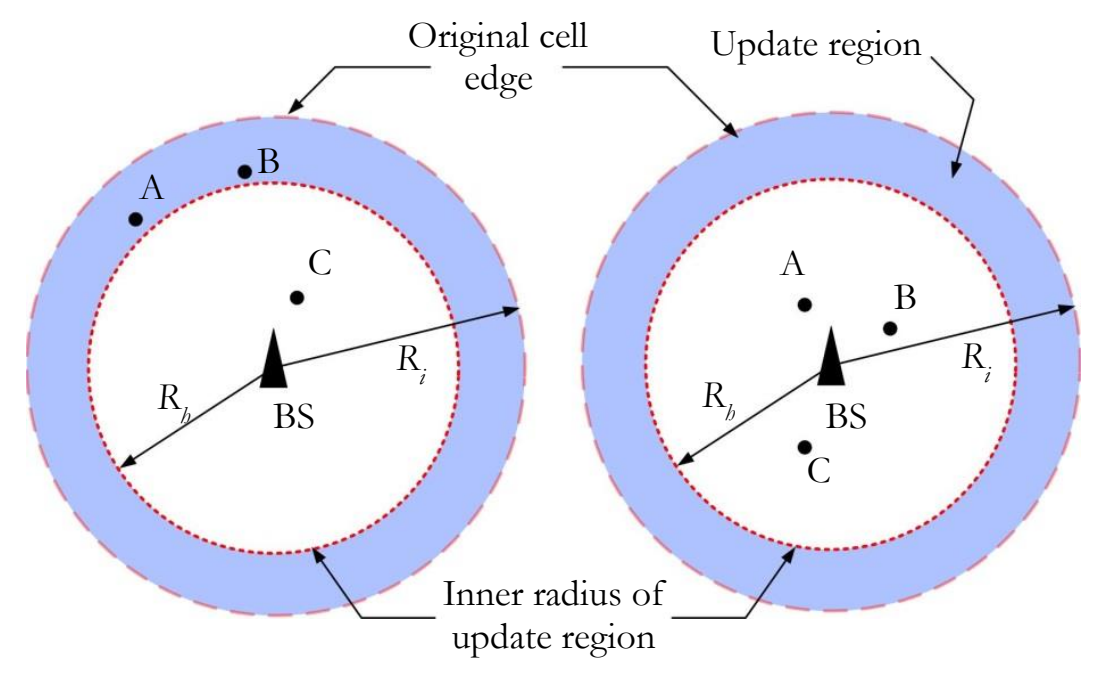

Situation I

Situation II

(a)

\section{For BS (network)}

- Set a uniform and constant $\Delta t_{u p} \forall$ MSs

- Reset $R_{i}$ from zoomed cell edge $\left(R_{z}\right)$ update process

- Receive $d_{M S}$ from MSs in specified update region during update process If no update message (because $\forall d_{M S}<R_{b}$ ) end Broadcast to $\forall$ MSs to perform update

- Find $\max \left[d_{M S}\right]$

- Perform cell zooming by setting $R_{z}=\max \left[d_{M S}\right]$ for specific update interval

- Broadcast information of current zoomed cell edge to $\forall$ MSs to evaluate update region in next period

\section{For MS}

- Listen to the BS to know inner bound $\left(\lambda_{b}\right)$ to sense update region

- Measure $d_{M S}$ by means of pilot signal strength

- When update time arrives

$$
\text { If } \mathrm{R}_{b} \leq d_{M S} \leq \mathrm{R}_{i}
$$

Perform location update

else

Skip location update

end

(b)

Fig. 3. Location-adaptive periodic update (LAPU): (a) conceptual demonstration; (b) pseudo code of LAPU. 


\section{Simulation Methodology}

\subsection{Simulation Models}

For the simulation of continuous cell zooming algorithm with different location update schemes (CPU, TAPU and LAPU), Poisson distribution is selected for the simulations of traffic arrival to the network and Random Waypoint model is used for the simulation of user mobility in the network. These can be seen in our previous work [29]. In Random Waypoint model, a random number of users move to random direction at each time. The movement of each user is independent of other users. The minimum pause time of moving users in Random Waypoint model is $1 \mathrm{~min}$.

Here, the maximum possible number of moving users at each time is justified with time dependent user mobility levels in a day that has been shown in Fig. 2(a) to create more realistic mobility behavior in simulation. Thus, time-dependent mobility level and movement speed of users taken are described in Table 1. The mobility level is the ratio of maximum possible number of moving users to the total number of users in the network. It means that the total moving users at a time will not be greater than maximum possible number of moving users at that time.

Table 1. Mobility level and moving speed range at different local hours [29].

\begin{tabular}{ccc} 
Local time $[\mathbf{h r}]$ & Mobility level & Movement speed range $[\mathrm{m} / \mathbf{s}]$ \\
\hline $24: 00<t \leq 05: 00$ & 0.1 & $0-50$ \\
$05: 00<t \leq 08: 00$ & 1.0 & $0-50$ \\
$08: 00<t \leq 11: 00$ & 0.5 & $0-50$ \\
$11: 00<t \leq 14: 00$ & 1.0 & $0-50$ \\
$14: 00<t \leq 16: 00$ & 0.5 & $0-50$ \\
$16: 00<t \leq 18: 00$ & 1.0 & $0-50$ \\
$18: 00<t \leq 24: 00$ & 0.5 & $0-50$ \\
\hline
\end{tabular}

\subsection{Network Parameters}

The performances of proposed location update schemes are evaluated in both omni-directional cell and sector-based cell networks because both types of cells are now in practical applications. Each network consists of seven cells in hexagonal arrangement.

Table 2. Simulation parameters of network $[9,34]$.

\begin{tabular}{clcc}
\hline Part & \multicolumn{1}{c}{ Parameters } & Omni-directional & Sector-based \\
\hline \multirow{5}{*}{ Base station } & Number of sectors & 1 & 3 \\
& Number of transceivers & SISO & SISO \\
& Original cell radius $\left(R_{i}\right)$ & $0.35 \mathrm{~km}$ & $0.35 \mathrm{~km}$ \\
& Maximum capacity & $0.4 \mathrm{Mb} / \mathrm{s}$ & $1.2 \mathrm{Mb} / \mathrm{s}$ \\
& Maximum transmitted power per sector $\left(P_{T \times i}\right)$ & $20 \mathrm{~kW}$ & $20 \mathrm{~kW}$ \\
\hline \multirow{2}{*}{$\begin{array}{c}\text { Network } \\
\text { traffic }\end{array}$} & Maximum number of traffic in each cell at & 250 voice calls & \multirow{2}{*}{750 voice calls } \\
& noon period (11:00-12:00) & \multicolumn{2}{c}{$12 \mathrm{~kb} / \mathrm{s}$} \\
& Data rate for single user (voice communication) & \multicolumn{2}{c}{$60 \mathrm{~s}$} \\
& Average sojourn time & \multicolumn{2}{c}{$360 \mathrm{~s}$} \\
\hline
\end{tabular}

The detailed parameters of the network taken in simulations are listed in Table 2. In an omnidirectional cell, there is only one sector and there are 3 sectors in a sector-based cell. Then single-inputsingle-output antennas are used in both cell types. The originally designed (maximum) cell radius is $0.35 \mathrm{~km}$ 
for both cell types. A maximum data rate of $0.4 \mathrm{Mb} / \mathrm{s}$ is provided by an omni-directional cell and $1.2 \mathrm{Mb} / \mathrm{s}$ by a sector-based cell [34]. The power required to transmit maximum data rate to the original designed cell edge is $20 \mathrm{~kW}$ in each sector. Although there can different types of communications (data, voice, video), only voice communication, which is dominant communication, is considered to simplify the simulation. For the users, the data rate required to make a voice call is $12 \mathrm{~kb} / \mathrm{s}$ and average duration (time taken) by each call is $60 \mathrm{~s}$ [9].

According to data rate for voice call and maximum capacity, 33 calls can be simultaneously served by an omni-directional cell and 100 calls by a sector-based cell at a time. The maximum numbers of calls (250 calls in omni-directional cell and 750 calls in sector-based cell) occur at midday (11:00-12:00). But it should be noted that this total phone calls are counted for one hour duration and only a certain number of phone calls arrive at a time and each call averagely lasts $60 \mathrm{~s}$. For example, for the calling rate of $250 \mathrm{calls} / \mathrm{hr}$, there are 25 calls at every 6 mins during one hour. Then a data rate of $(25$ calls x $12 \mathrm{~kb} /$ calls $) 0.3 \mathrm{Mb} / \mathrm{s})$ must be provided by each omni-directional cell at every interval.

\subsection{Simulation Scenarios}

Four different simulation scenarios are considered. These scenarios are constructed to attain understanding on the relationship between the performance of proposed update schemes and network related parameters.

Scenario 1: Firstly, the performance of proposed LAPU scheme is analyzed by changing the inner radius update region. The inner radius of update region in LAPU is varied in the range of $70-90 \% \mathrm{R}_{\mathrm{z}}$. In this scenario, the number of users in the network is 100 and the moving speed range is $[0-50] \mathrm{m} / \mathrm{s}$.

Scenario 2: In this scenario, the performances of proposed location update schemes are analyzed in a network with the variation of number of users. Thus, the moving speed range of users, [0-50] $\mathrm{m} / \mathrm{s}$, is kept constant and the number of users in the network is changed from 50 to 300.

Scenario 3: In the last scenario, the performances of proposed location update schemes are evaluated in a network with the variation of average moving speeds of users. Thus, the average moving speeds of users in the network is varied from $1 \mathrm{~m} / \mathrm{s}$ to $30 \mathrm{~m} / \mathrm{s}$ keeping the number of users in the network constant as 100 .

\subsection{Performance Metrics}

The performance metrics used to analyze and judge the performances of proposed adaptive location update schemes are transmitted power ratio, outage ratio and the number of update messages during cell zooming operation with continuous cell zooming algorithm.

Transmitted power ratio: The transmitted power ratio is evaluated as the ratio of transmitted power with cell zooming to peak conventional transmitted power as follows:

$$
\text { Transmitted power ratio }=\frac{\text { Transmitted power with cell zooming }}{\text { Peak. conventional transmitted power }}
$$

Outage ratio: Traditionally, outage is defined for a situation when a user experiences lower strength than receivable one [9]. Thus, in this work, the users out of convergence region during each cell zooming period are counted as outages. The outage ratio in a cell zooming period is the ratio of total number of users who undergo outage during a cell zooming period to the total number of users who exist in the network.

$$
\text { Outage ratio }=\frac{\text { Total number of outage users in a cell zooming period }}{\text { Toal number of users in the network }}
$$

Number of update messages: The number of update messages, which represents the signaling cost, exchanged between the BSs and users is evaluated in every cell zooming period.

\section{Results and Discussion}

\subsection{Cell Zooming in Omni-directional and Sector-based Cell Networks}

The simulations are performed in MATLAB software. The results are average values of 3 days' operation. In conventional periodic update (CPU) scheme and location-adaptive periodic update (LAPU) scheme, the constant and uniform update interval of 2 minutes, is set for all users and for the whole day. In timeadaptive periodic update, $\Delta t_{u p p}, \Delta t_{u p 2}$ and $\Delta t_{u p 3}$ are set as 5 minutes, 3 minutes and 2 minutes respectively. 
Here, to lower the signalling cost at low mobility levels, some update intervals in TAPU is set to the longer values to reduce the signalling messages compared to CPU.

Figures 4(a)-(b) show a sample distribution of MSs in each network and configurations of omnidirectional and sector-based cells under zoomed condition according to the location of the farthest MS in each cell. It can be seen that cell zooming is performed cell by cell in omni-directional cell network because it has only one sector. Meanwhile, in sector-based cell network, cell zooming is performed sector by sector based on the farthest user in each sector. It is understandable that cell zooming can be performed more significantly in the sector-based cell network compared to omni-directional cell network. In other words, the zooming in sector-based cell is more effective than that in omni-directional cell.

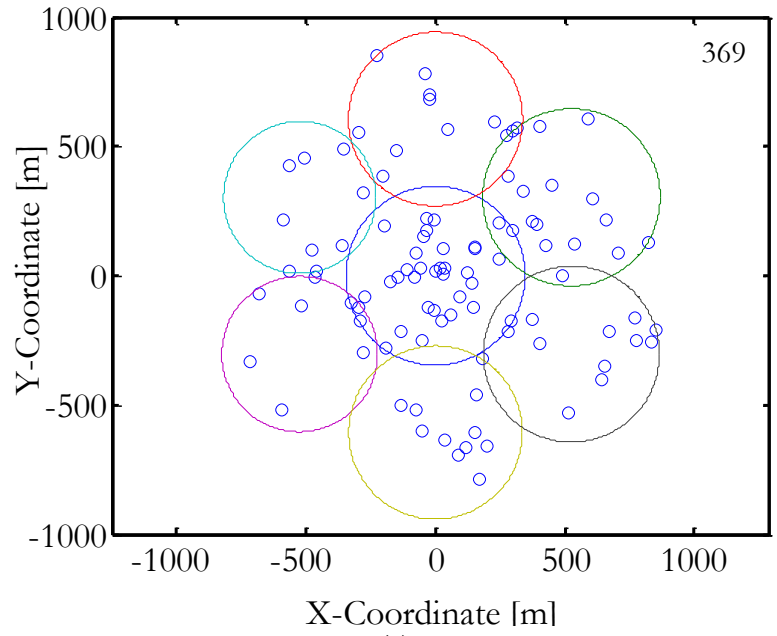

(a)

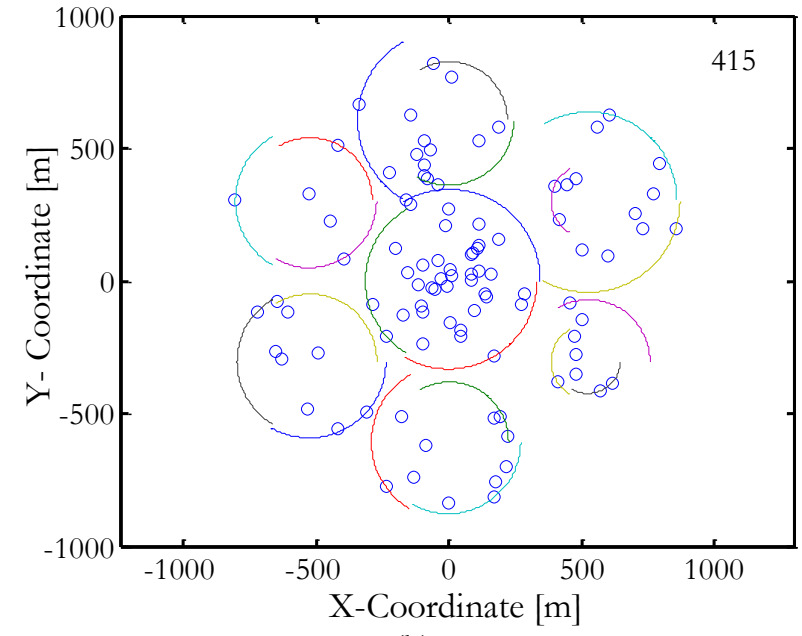

(b)

Fig. 4. Distribution of MSs and configuration of zoomed cells: (a) Omni-directional cell network; (b) Sector-based cell network

\subsection{Performance of LAPU Scheme with Different Inner Radiuses of Update Region}

It has been previously explained that in LAPU scheme, the inner radius of update region for coming update process is set with respect to zoomed cell edge in current period. This scenario is conducted to analyze the performance of LAPU scheme with different inner radiuses of update region. It is changed from $50 \%$ to $90 \%$ of zoomed cell radius.

Figures 5(a)-(b) show the comparison of transmitted power ratios in continuous cell zooming algorithm with LAPU scheme with different $R_{b} / R_{z}$ ratios. It can be seen that the transmitted power ratio increases with the increment of inner radius. In other words, the transmitted power ratio becomes larger when the size of update region becomes smaller. However, the variation of transmitted power ratio with the increment of inner radius of update region is very small. In this scenario, the number of users, the moving speed range, and the update interval are the same. Thus, it can be understood that the increment of transmitted power ratio is not because of cell zooming condition. This very slight increment is induced by power consumption required in broadcasting to all users to perform update when there is no user in update region (Situation II) which is demonstrated in Fig. 3(a). The situation II can often occur when the size of update region is small and the number of users is also small. On the other hand, when update region size is lager, it can increase the number of update messages. Thus, a careful selection of update region size is required for a certain number of users in the cell. 


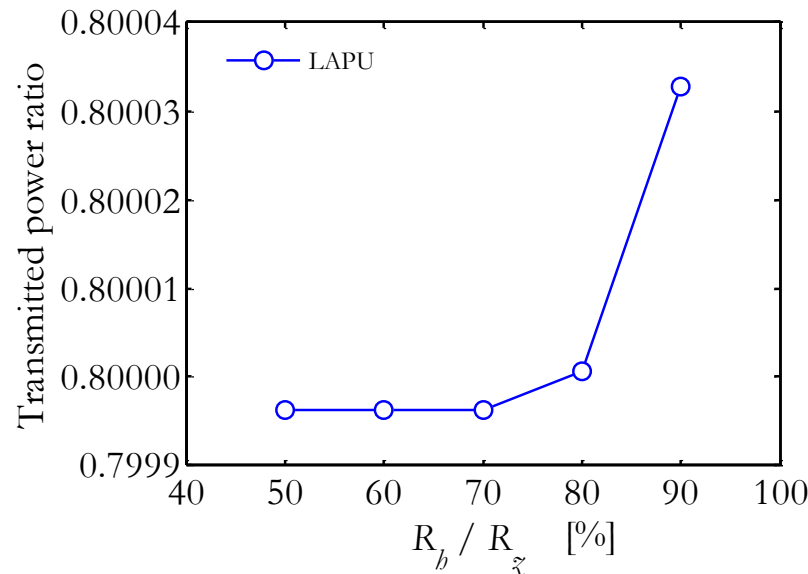

(a)

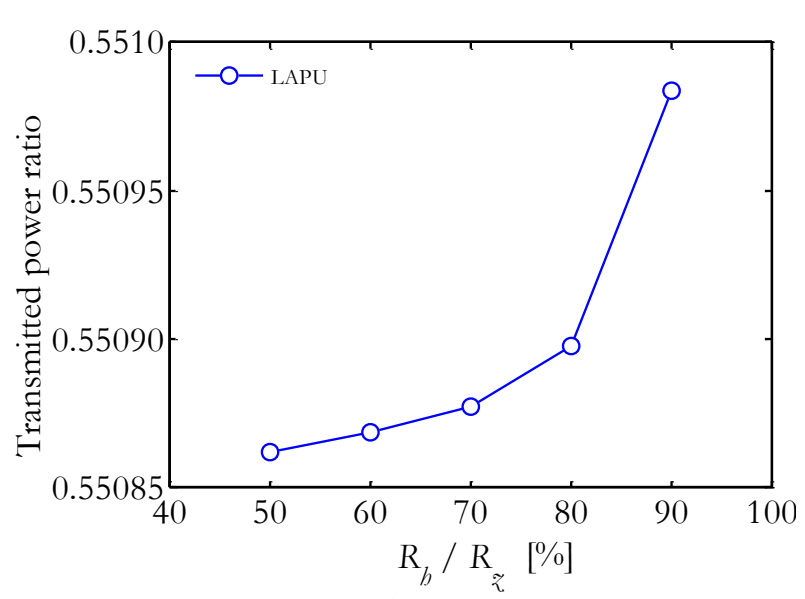

(b)

Fig. 5. Transmitted power ratios in cell zooming with LAPU scheme: (a) omni-directional cell network; (b) sector-based cell network.

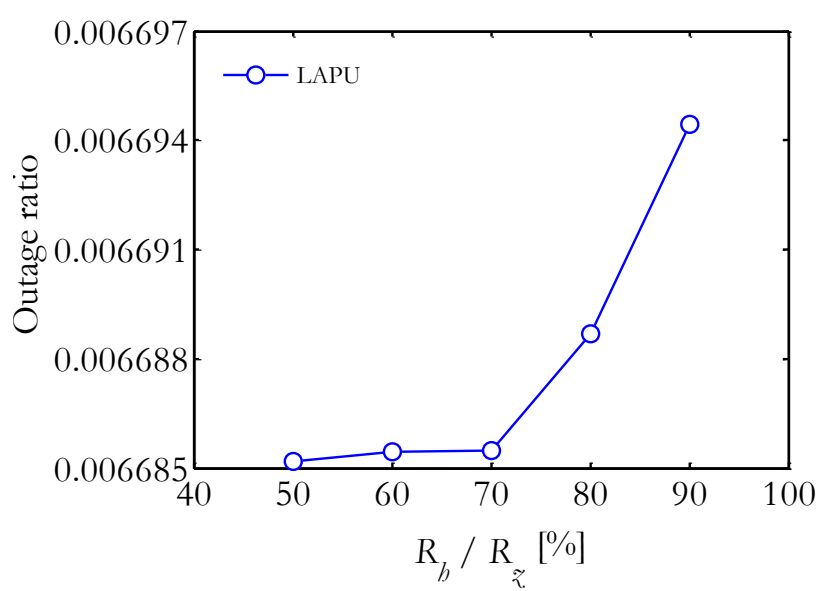

(a)

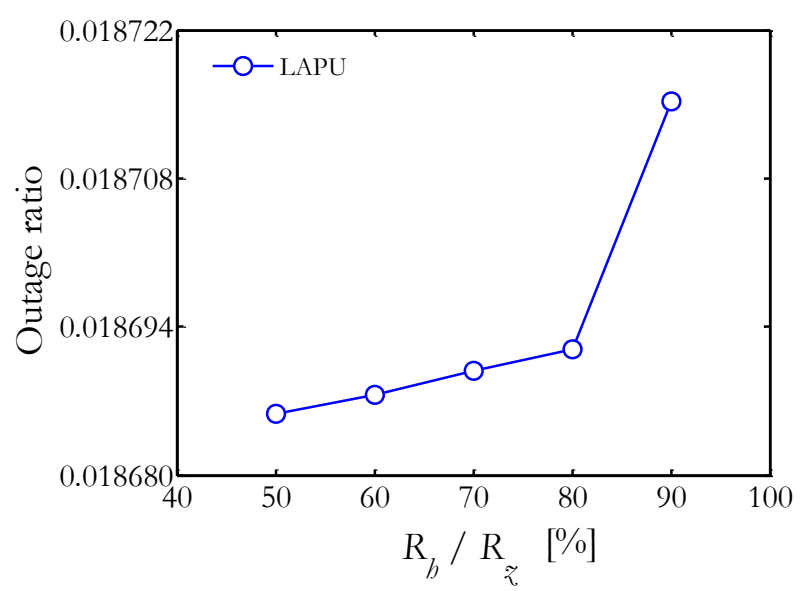

(b)

Fig. 6. Outage ratios in cell zooming with LAPU scheme: (a) omni-directional cell network; (b) sectorbased cell network.

Then, the comparison of outage ratios occurred in cell zooming operation is shown in Figs. 6(a)-(b). In both omni-directional and sector-based cell networks, it shows an increasing trend with the increment of inner radius of update region although the difference among values is not significant. It can be explained by giving the following consequential facts. According to the trend of transmitted power ratio, it illustrates that when update region size becomes smaller, the Situation II in LAPU scheme (in Fig. 3(a)) more often happens. It explains that the users distribute near the BS rather than at cell edge. It is true because when the number of users is small, the probability of existence of user near cell edge is small compared to a condition with a large number of users. Thus, transmission range can be reduced more effectively. When cell zooming is effective in on one hand, the outage area is also large on the other hand (see in Fig. 1). Thus, the moving users can undergo outage more easily. The other reason is that when update area is small, it is not easy to capture the locations of more frequently moving users in the cell.

Figures 7(a)-(b) depict the number of update messages in LAPU scheme with various inner radius of update region. It is clear the number of update messages will be reduced when the size of update region becomes smaller according to LAPU concept. It almost linearly decreases with the increment of $R_{b} / R_{i}$ in both omni-directional and sector-based cell network. According to the results in the transmitted power outage ratio and number of update messages, the $\mathrm{R} h / \mathrm{R} z$ of $70 \%$ is suitable for 100 users in the network. However, it should be noted that optimum update region size can vary according to the number of user in the network. In this work, to measure performance of LAPU algorithm, $R h / R_{z}$ is set to $70 \%$. 


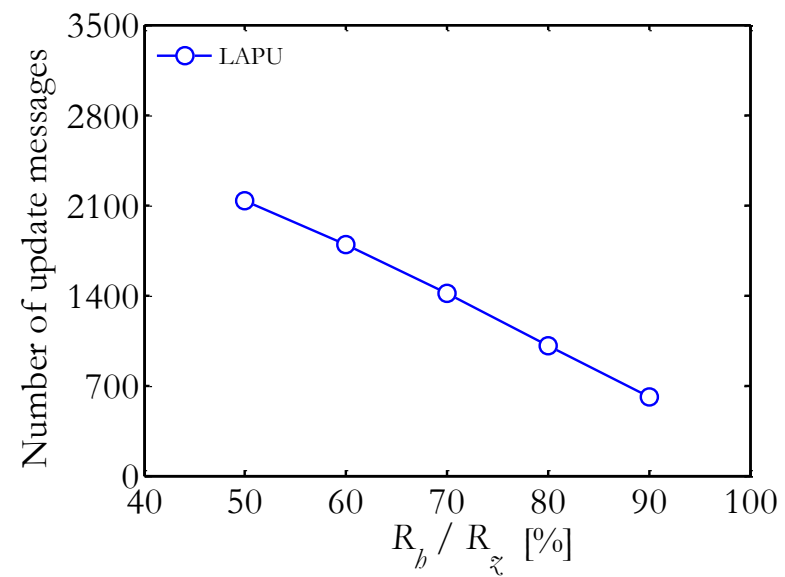

(a)

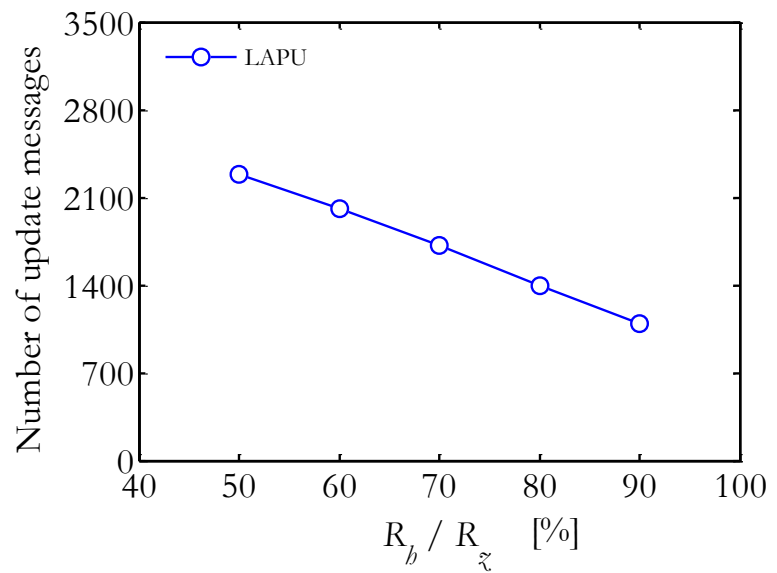

(b)

Fig. 7. Numbers of update messages in cell zooming with LAPU scheme: (a) omni-directional cell network; (b) sector-based cell network.

\subsection{Performances of Proposed Location Update Schemes in a Network with Different Numbers of Users}

In this scenario, the performances of TAPU and LAPU are analyzed in the network with the variation of number of users. The result at each point is the average value of the whole day operation which is averaged from 3 day's operation. Figures 8(a)-(b) show the comparison of transmitted power ratios given by continuous cell zooming algorithm with three different update schemes at numbers of users.

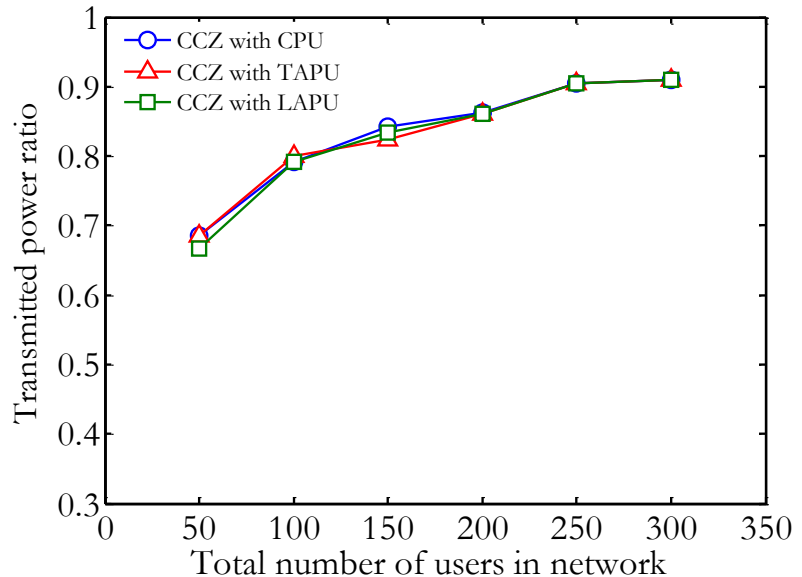

(a)

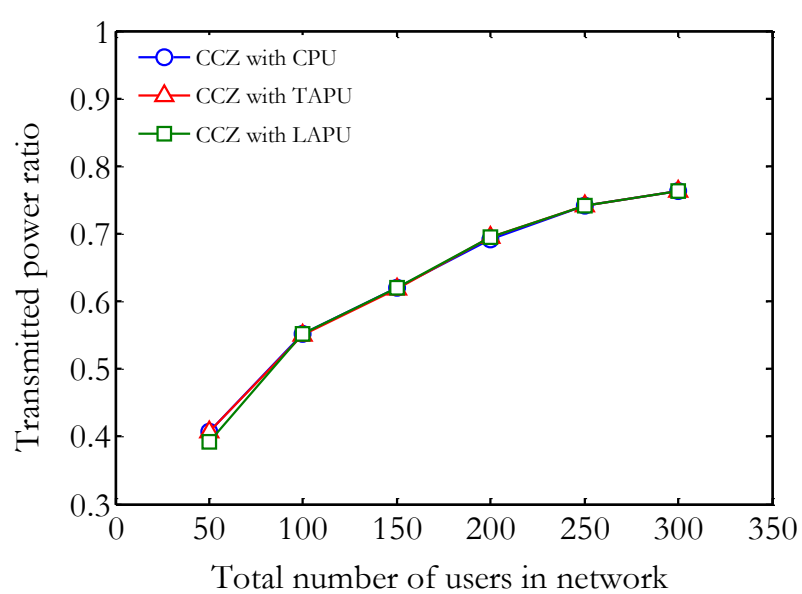

(b)

Fig. 8. Transmitted power ratios in cell zooming at different numbers of users: (a) omni-directional cell network; (b) sector-based cell network.

The transmitted power ratio increases with the increment of number of users in the network. In other words, power saving decreases with the increment of the number of users. In omni-directional cell network, the power saving is about $35 \%$ when the number of users is 50 . When the number of users is increased to 300 , the power saving is about $10 \%$. Meanwhile, it is about $61 \%$ when the number of users is 50 and $24 \%$ when the number of user is 300 in sector-based cell network. The reason can be explained as follows. When there are a large number of users, there is a larger probability of existence of user near cell edge. Thus, cell zooming cannot be performed very significantly in a condition with a large number of user compared to a condition with a smaller number of users. 


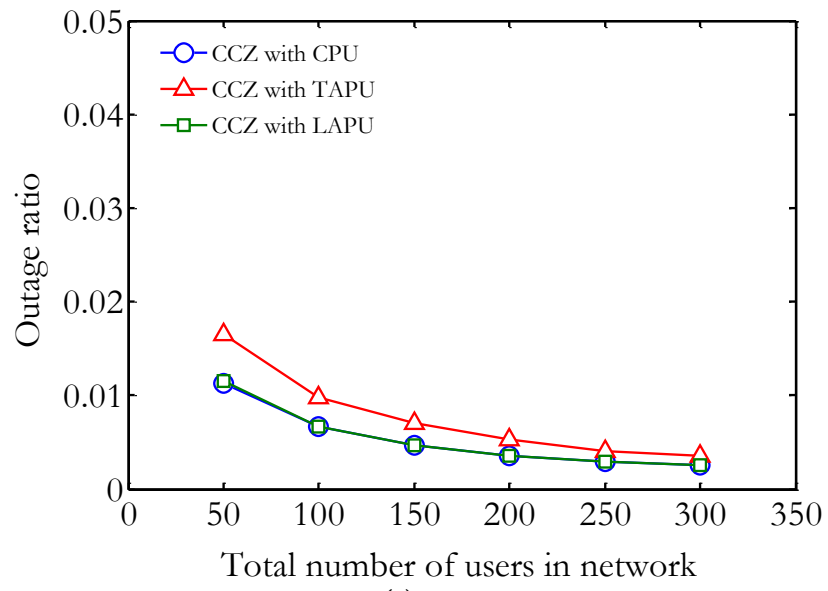

(a)

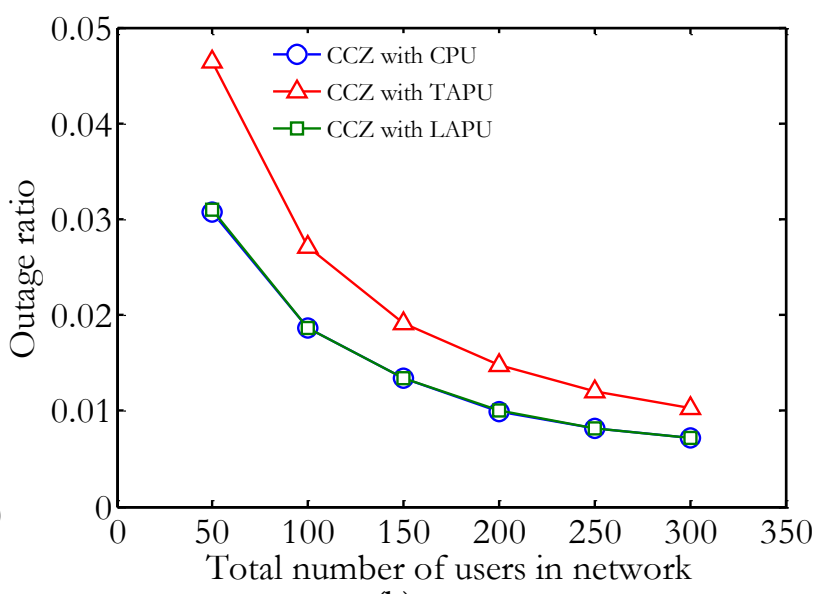

(b)

Fig. 9. Outage ratios in cell zooming at different numbers of users: (a) omni-directional cell network; (b) sector-based cell network

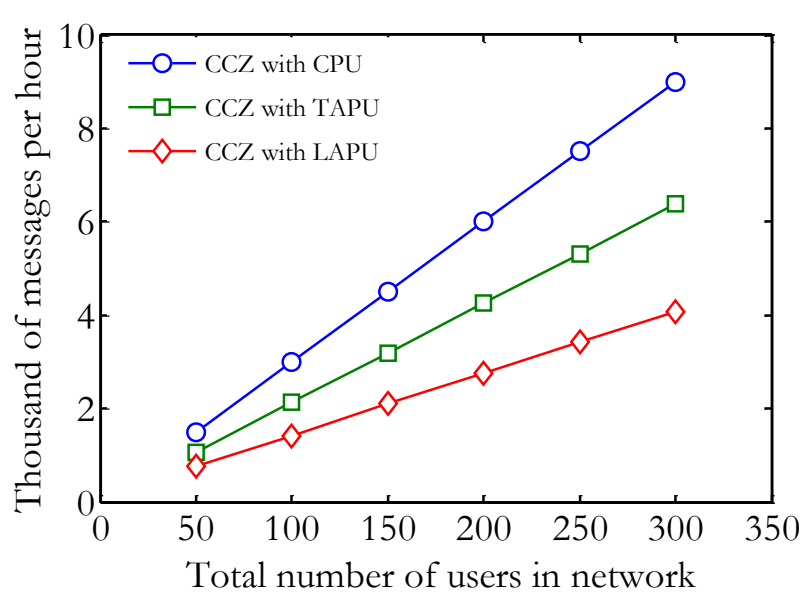

(a)

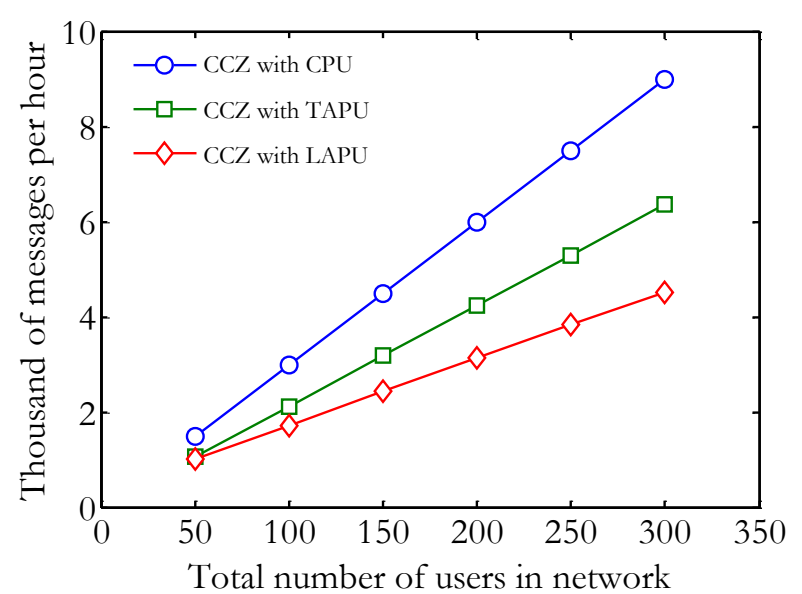

(b)

Fig. 10. Number of update messages in cell zooming at different numbers of users: (a) omni-directional cell network; (b) sector-based cell network.

The comparisons of outage ratios occurred in CPU, TAPU and LAPU schemes in omni-directional cell network and sector-based network are shown in Figs. 9(a)-(b). It can be seen that the outage ratios in CPU and LAPU are kept approximately the same. The reason is that update intervals are the same in these two schemes. The outage ratios in TAPU are higher than that in CPU and LAPU in both omni-directional cell network and sector-based cell network. The outage ratio in each scheme decreases with the increment of the number of users in the network. There are two reasons. The reasons can be explained as follows. The first reason is that when the number of users in the cell is small, there is less possibility of existence of user near the cell edge. Therefore, the cell can be zoomed considerably and the outage area is also large. In this condition, outage easily occurs. In contrast, the larger the number of users, the higher the probability of existence of users near the cell edge. It gives less chance to zoom the cell or the cell can be zoomed only a few percent. Therefore, the outage area during cell zooming is smaller. As a result, outage ratio decreases when the number of users in the network increases. There is another reason of decreasing outage ratio. One should remember that outage ratio is the ratio of total outage users to the total users existed in the network (as defined in Eq. (3)). According to this definition, outage ratio will decrease when total number of users, which is denominator, increases. The outage ratio is larger in sector-based cell network. As explained previously, the cell is zoomed more significantly in sector-based cell network. It makes larger outage area in sector-based cell network, which results in a larger number of outages.

Figures 10(a)-(b) depict average number of update messages per hour given by three update schemes in omni-directional cell network and sector-based cell network. Firstly, one can understand that average number of update messages in CPU, TAPU and LAPU linearly increases with the increment of the number of users in the network. Thus, it is clear that the number of update messages is obviously dependent of the 
number of users and update interval. It is obvious that LAPU schemes give better performance in reducing the number of update messages. The performance of TAPU is slightly lower than LAPU. LAPU gives approximately the same performance in omni-directional cell network and sector-based cell network. The difference is just a few hundreds of messages.

\subsection{Performances of Proposed Location Update Schemes in a Network with Different Average Moving Speeds of Users}

The performances of proposed update schemes depend on the number of users and user distribution in the network. In turn, the user distribution can be influenced by moving speed of users at a constant number of users in the network. Therefore, in this scenario, the relationship between the performances of proposed update schemes and average moving speed of users is analyzed. The number of users in the network is kept constant as 100. Then average moving speed is changed from $1 \mathrm{~m} / \mathrm{s}$ to $30 \mathrm{~m} / \mathrm{s}$.

Figures 11(a)-(b) describe the transmitted power ratios in continuous cell zooming algorithm with three different update schemes. Here, it can be observed that the transmitted power ratios in both omnidirectional and sector-based cells has no consistent increasing of decreasing trend with the increment of average moving speed of users. It can be said the trend is dynamic since there are increasing trend and decreasing trend. Also, it is noticeable that the change of transmitted power ratio is not significant. It changes in the range of $0.7-0.8$ in omni cell network and in the range of $0.4-0.6$ in sector-based cell network. The reasons can be explained by using the following demonstrations.

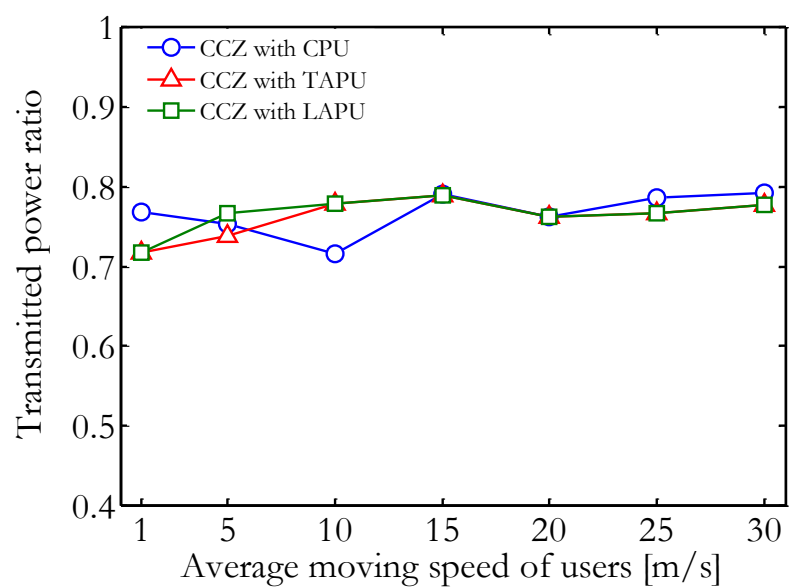

(a)

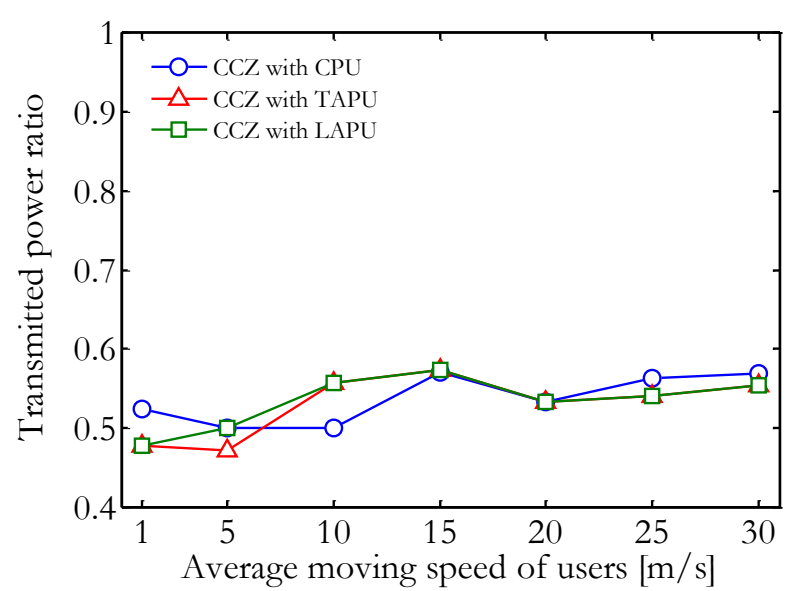

(b)

Fig. 11. Transmitted power ratios in cell zooming at different average speeds: (a) omni-directional cell network; (b) sector-based cell network.

Figures 12(a)-(d) show the possible user positions and moving directions with a high speed. Figure 12(a) shows that the users at the cell center can reach to the cell edge very easily due to moving with a high speed. In the same way, Fig. 12(b) demonstrates that the users at the cell edge can reach to cell center very easily due to moving with a high speed. Fig. 12(c) displays that users can move in and move out very dynamically in the cell area. Therefore, it is not certain to state that the transmitted power will be higher or lower due to moving with a higher speed. It depends on where the users start to move and how the users move.

Then, one may wonder if transmitted power will be low if the moving speed is low. It is also not certain. As shown in Fig. 12(d), when users are moving very slowly, the user at the cell edge cannot reach very quickly to the cell center. It will maintain its far distance for long. In the same way, the user at cell center will not go far very easily. It can maintain its close distance to BS for a long time. According to these two conditions, the transmitted power can be higher or can be lower. Thus, the transmitted power ratio with the variation of average moving speed has no consistent increasing or decreasing trend. 


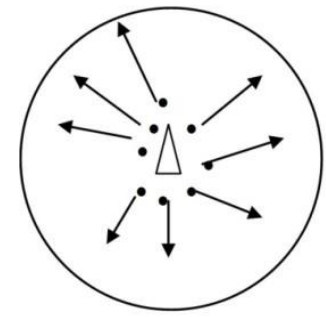

(a)

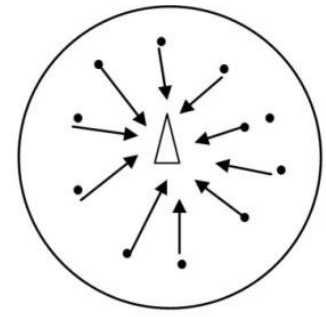

(b)

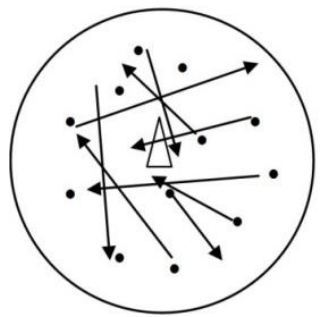

(c)

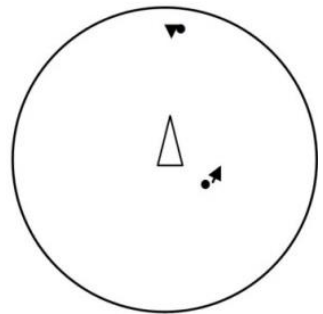

(d)

Fig. 12. (a)-(d) Possible user moving conditions in a cell.

The comparison of outage ratios in cell zooming due to three different update schemes is shown in Figs. 13(a)-(b). The outage ratio in TAPU is the largest and average outage ratios in CPU and LAPU are still approximately the same. For each update scheme, the effect of speed on outage ratio is obvious. It is clear that the outage can easily occurs when the users are moving with a very high speed. Thus, the outage ratio increases with the increment of average speed. The outage ratio is higher in sector-based cell network.

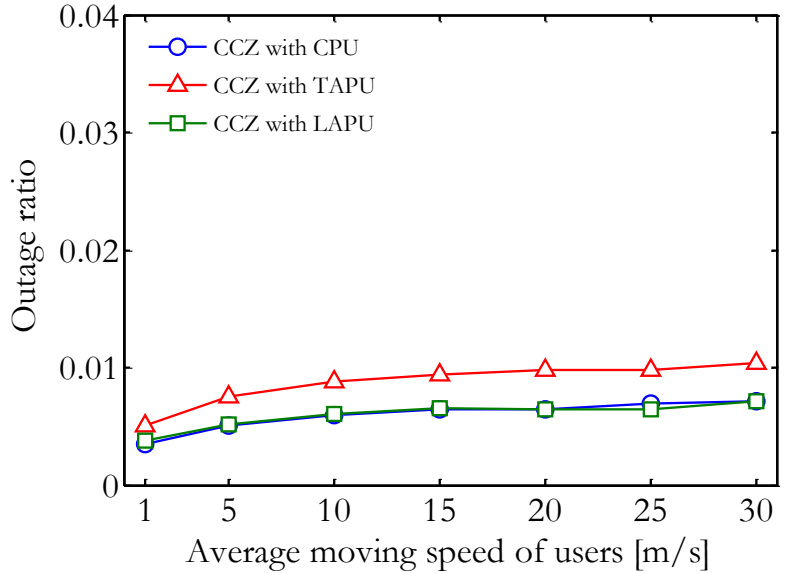

(a)

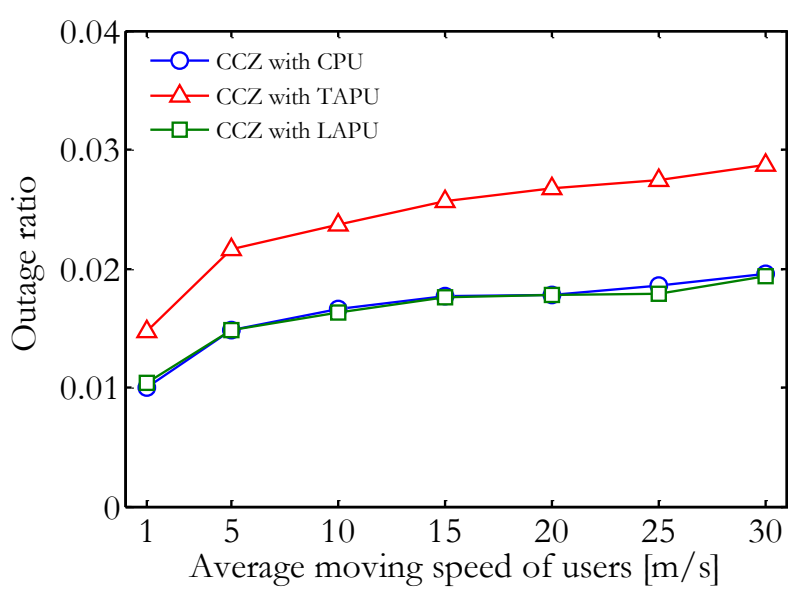

(b)

Fig. 13. Outage ratios in cell zooming at different average speeds: (a) omni-directional cell network; (b) sector-based cell network.

The number of update messages in LAPU depends on how many users exist in the update region. In other words, it also depends on how the users move, from cell center to cell edge or from cell edge to cell center. According to the results shown in Figs. 14(a)-(b), the number of update messages in LAPU has the same trend with the transmitted power ratio in LAPU. When the transmitted power is low, it means that the user move in. Thus, there will be less number of users in update region, which results in lower number of update messages. When the transmitted power is high, it means that the users move to cell edge. Thus, there will be a larger number of users in update region, which results in a larger number of update messages. LAPU gives a slightly better performance in omni-directional than in the sector-based network. Meanwhile CPU and TAPU have constant average number of update messages at each hour in a day. Here, it should be noted that in each scheme in practice, there could be some users who miss to update due to moving with a very high speed. 


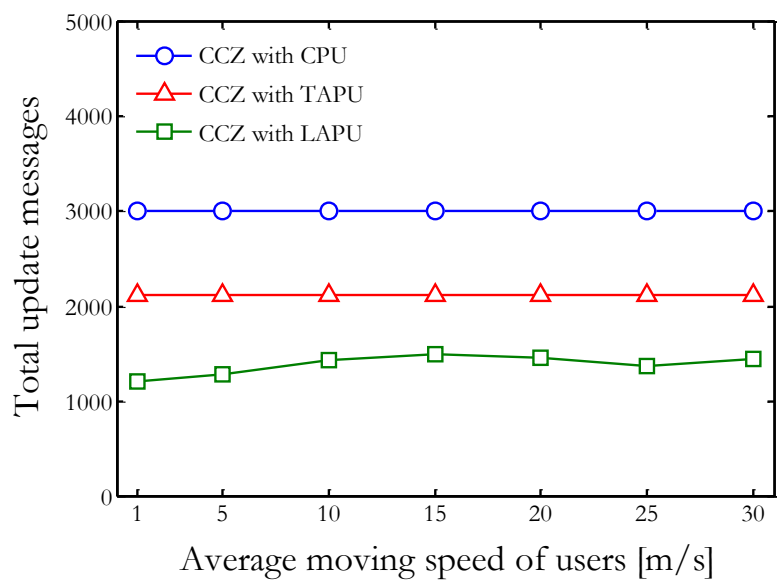

(a)

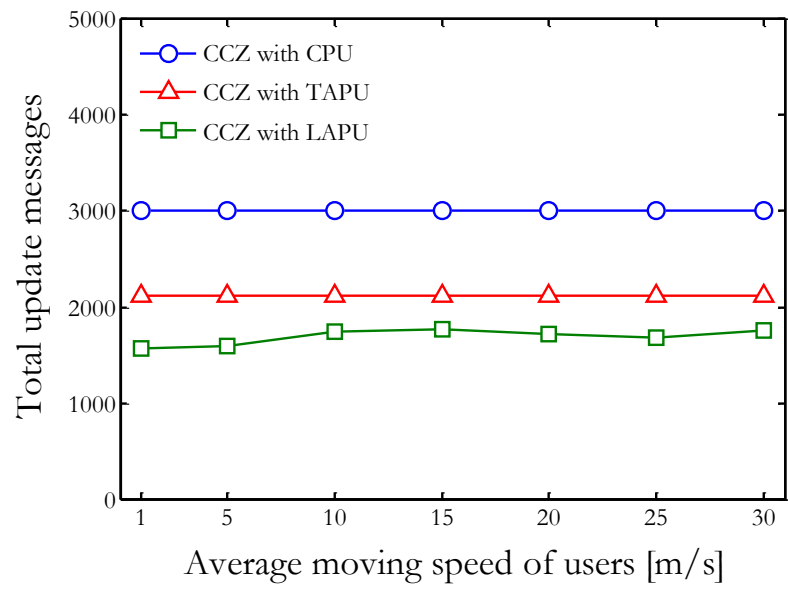

(b)

Fig. 14. Number of update messages in cell zooming at different average speeds: (a) omni-directional cell network; (b) sector-based cell network.

\section{Conclusion and Recommendation}

In this work, the performances of two location update schemes, namely time-adaptive periodic update (TAPU) and location-adaptive periodic update (LAPU), are evaluated in different scenarios. The location update schemes are applied in continuous cell zooming algorithm in mobile wireless networks. The performances of these two schemes are compared with that of conventional periodic update (CPU) scheme. After analyzing the performances of TAPU and LAPU in different scenarios in both omni-directional and sector-based cell networks, the following conclusion can be drawn.

Compared to CPU, the proposed location update schemes (TAPU and LAPU) have no significant effect on power saving capability of continuous cell zooming algorithm. In daily operation the length of update interval applied in proposed update schemes (TAPU and LAPU) will affect the outage. The scheme with a longer update interval will give a larger number of outages and the scheme with a smaller update interval will give a smaller number of outages. On the other hand, the smaller the update interval in CPU and TAPU, the larger the number of update messages in cell zooming operation which is unfavorable. In this sense, LAPU is favorable because it can reduce the number of update messages depending on the size of update area specified.

In any scheme, the transmitted power ratio increases, the outage ratio decreases and the number of update messages increases with the increment of the number of users. The average moving speed of users in the network does not show consistent effect on power saving capability and the number of update messages. However, the outage becomes higher when the average moving speed is higher.

In terms of power saving, the proposed update schemes perform better in sector-based cell network. However, the performances of TAPU and LAPU are better in omni-directional cell network in terms of outage ratio and the number of update messages.

The information in this work is still limited and the work is still simple. For example, a learning program for user mobility level in a day in a network can be implemented rather than assuming it. Then, a new update method can be developed by combining currently proposed update methods (TAPU and LAPU). These can be recommended as further works.

\section{References}

[1] A. Amanna. (2010). Green Communications: Annotated Literature Review and Research Vision. [Online]. Available: http://www.learningace.com

[2] Y. Zhao and Y. Hao, "A subject-specificity analysis of radio channels in wireless body area networks," Eng J, vol. 15, pp. 39-48, 2011.

[3] M. Sivakumar and M. K. Jayanthi, "Reliability analysis of link stability in secured routing protocols for MANETs,” Eng J, vol.18, pp. 65-76, 2013.

[4] A. Nirapai and S. Chantaraskul, "Centralized control for dynamic channel allocation in IEEE 802.15. 4 based wireless sensor networks," EngJ, vol. 18, pp. 151-164, 2014. 
[5] N. Zhisheng, W. Yiqun, G. Jie, and Y. Zexi, "Cell zooming for cost-efficient green cellular networks," IEEE Commu Mag, vol. 48, pp. 74-79, Nov., 2010.

[6] K. C. Tun and K. Kunavut, "An overview of cell zooming algorithms and power saving capabilities in wireless networks," KMUTNB: Int J Appl Sci Tec, vol. 7, no. 3, pp. 1-13. Aug., 2014.

[7] Oh. Eunsung, B. Krishnamachari, L. Xin, and N. Zhisheng, "Toward dynamic energy-efficient operation of cellular network infrastructure," IEEE Commu Mag, vol. 49, pp. 56-61, Jun., 2011.

[8] V. Prithiviraj, S. B. Venkatraman, and R. Vijayasarathi, "Cell zooming for energy efficient wireless cellular network," J Green Eng, vol. 3, pp. 421-434, Sept., 2013.

[9] S. Kokkinogenis and G. Koutitas, "Dynamic and static base station management schemes for cellular networks," in Proc. Global Communications Conference, GLOBECOM, 2012, pp. 3443-3448.

[10] L. Suarez, L. Nuaymi, and J.-M. Bonnin, "Analysis of the overall energy savings achieved by green cellbreathing mechanisms," in Proc. Sustainable Internet and ICT for Sustainability (SustainIT), 2012, pp. 1-6.

[11] G. Li, S. Jin, F. Zheng, X. Gao, and X. Wang,"Dynamic base station sleeping scheme for potential super density networks," in Proc. International Conference on Wireless Communications \& Signal Processing (WCSP), 2013, pp. 1-5.

[12] R. Balasubramaniam, S. Nagaraj, M. Sarkar, C. Paolini, and P. Khaitan, "Cell zooming for power efficient base station operation," in Proc. the 9th International Conference on Wireless Communications and Mobile Computing (IWCMC), 2013, pp. 556-560.

[13] K. C. Tun, and K. Kunavut, "Performance evaluation of dynamic cell zooming algorithms in omnidirectional and sector-based cells," in Proc. International Joint Conference on Computer Science and Software Engineering (JCSSE), 2014, pp. 158-163.

[14] D. Senzaki, G. Chakraborty, H. Mabuchi and M. Matsuhara, "Mobility pattern learning and route prediction based location management in PCS network," in Proc. 20th International Conference on Advanced Information Networking and Applications (AINA), 2006, pp. 5-10.

[15] J. S. M. Ho and J. Xu, "History-based location tracking for personal communications networks," in Proc. 48th IEEE Vebicular Technology Conference (VCT98), 1998, pp. 244-248.

[16] K. T. Chen, S. L. Su, R. F. Chang, "Design and analysis of dynamic mobility tracking in wireless personal communication networks," IEEE T Veh Commun, vol. 51, no. 3, pp. 486-497, May., 2002.

[17] M. S. Sricharan and V. Vaidehi, "A Dynamic distance based location management strategy utilizing user profiles for next generation wireless networks," in Proc. First International Conference on Industrial and Information Systems, 2006, pp. 388-392.

[18] P. P. Gregory and I. L. Chih, "A profile-based location strategy and its performance," IEEE J Sel Area Comm, vol. 15, no. 8, pp. 1415-1424, Oct.,1997.

[19] M. Wencha and F. Yuguang, "A user mobility-pattern-based location strategy for next generation wireless multimedia networks," Global Telecommunications Conference (GLOBECOM '03), 2003, pp. 30983102.

[20] Z. Yi-hua and V. C. M. Leung, "Optimization of distance-based location management for PCS networks," IEEE T Wirel Commu, vol. 7, no. 9, pp. 3507-3516, Sep., 2008.

[21] C. K. Ng and H. W. Chan, "Enhanced distance-based location management of mobile communication systems using a cell coordinates approach," IEEE T Mobile Comput, vol. 4, no. 1, pp. 41-55, Jan.-Feb., 2005.

[22] L. Keqin, "Analysis of distance-based location management in wireless communication networks," IEEE T Parall Distr, vol. 24, no. 2, pp. 225-238, Feb., 2013.

[23] Z. Yi-hua and V. C. M. Leung, "Optimization of sequential paging in movement-based location management based on movement statistics," IEEE T Veh Technol, vol. 56, no. 2, pp. 955-964. Mar., 2007.

[24] Z. Qinglin, S. C. Liew and Y. Yao, "Location update cost of distance-based scheme for PCS networks with CTRW model," IEEE Commu Lett, vol. 13, no. 6, pp. 408-410, Jun., 2009.

[25] U. Madhow, M L. Honig and K. Steiglitz, "Optimization of wireless resources for personal communications mobility tracking," IEEE/ ACM T Networking, vol. 3, no. 6, pp. 698-707, Dec., 1995.

[26] R. M. Rodriguez-Dagnino, H. Takagi, "Movement-based location management for general cell residence times in wireless networks," IEEE T Veh Technol, vol. 56, no. 5, pp. 2713-2722, Sept., 2007.

[27] Z. Naor and H. Levy, "Minimizing the wireless cost of tracking mobile users: an adaptive threshold scheme," in Proc. IEEE Seventeenth Annual Joint Conference of the IEEE Computer and Communications Societies (INFOCOM '98), 1998, pp. 720-727. 
[28] S. K. Shah, S. Tekinay and C. Saraydar, , "Co-operative location update algorithm for mobiles in next generation cellular networks," Workshop on High Performance Switching and Routing (HPSR), 2004, pp. 331-336, 2004.

[29] K. C. Tun and K. Kunavut, "Adaptive location update schemes for continuous cell zooming algorithm in wireless networks," in Proc. the $7^{\text {th }}$ International Conference on Knowledge and Smart Technology (KST 2015), 2015, pp. 155-160.

[30] I. Siomina, P. Värbrand, and D. Yuan, "Pilot power optimization and coverage control in WCDMA mobile network," Int J Manage Sci, vol. 35, pp. 683-696, 2007.

[31] P. Värbrand, and D. Yuan, "A mathematical programming approach for pilot power optimization in WCDMA networks," in Proc. the Australian Telecommunications, Networks and Applications Conference (ATNAC'03), 2003.

[32] K. Valkealahti, A. Hoglund, J. Parkkinen and A. Flanagan, "WCDMA common pilot power control with cost function minimization," in Proc. the 56th IEEE vehicular technology conference, 2002, pp. 2244 2247.

[33] L. Zhao, G. Yao and J. W. Mark, "Mobile positioning based on relaying capability of mobile stations in hybrid wireless networks," IEEE Pro Commu, vol. 153, no. 5, pp. 762-770, Oct., 2006.

[34] M. Deruyck, E. Tanghe, W. Joseph and L. Martens, "Modelling and optimization of power consumption in wireless access networks," Comput Commun, vol. 34, pp. 2036-2046, Apr., 2011. 